For Internal Distribution Only

Accelerator Division

Alternating Gradient Synchrotron Department

BROOKHAVEN NATIONAL LABORATORY

Upton, New York 11973

Accelerator Division

Technical Note

AGS/AD/Tech. Note No. 466

Heavy Ion Formulation

S.Y. Zhang

July 29, 1997 


\section{Heavy Ion Formulation}

Let the heavy ion parameters be developed using the unit per charge, then the formulations available for electrons and protons can be directly used. In such a way, a heavy ion is treated as a group of pseudo-particles. Each pseudo-particle has its rest mass, the energy, the momentum, and other parameters as well, depending not only on the atomic mass but also on the charge state.

The use of the unit per charge for the heavy ion formulations is based on the fact that the longitudinal and transverse focusing is determined directly by the unit per charge.

Once the charge state is changed, the parameters per charge would experience discontinuous transitions. Because of this reason, the energy and momentum per nucleon are often used for convenience. For the same reason, the longitudinal emittance per nucleon is also used. These parameters can be easily obtained from the unit per charge.

To conserve the longitudinal emittance, an RF matching is needed at the charge state change, since that the longitudinal focusing will be changed. This situation is different from the adiabatic $\mathrm{RF}$ voltage change, it will be similar to, but not the same as, the harmonic number change.

In this note, we will go through with main heavy ion formulations. For illustration, the examples of the Gold beam $A u^{77+}$, presently operated at the AGS, will be used.

\section{Fundamental Parameters}

We start from the heavy ion rest mass,

$$
m_{0}=A u-Q m_{e 0}
$$

where $A$ is the atomic mass, 196.97 for Gold, $Q$ is the charge state, 77 presently at the AGS, $u$ is the unified atomic mass unit, $0.9315 \mathrm{GeV} / \mathrm{c}^{2}$, and $m_{e 0}$ is the rest mass of electron, 0.511 $M e V / c^{2}$. The rest mass and energy per charge are defined as,

$$
m_{0, c h}=\frac{m_{0}}{Q}
$$

and,

$$
E_{0, c h}=m_{0, c h} c^{2}
$$

The momentum and energy per charge are, 


$$
p_{c h}=m_{0, c h} c \beta \gamma
$$

and,

$$
E_{c h}=E_{0, c h} \gamma
$$

The pseudo-particle classical radius is

$$
r_{0, c h}=\frac{1}{4 \pi \epsilon_{0}} \frac{e^{2}}{m_{0, c h} c^{2}}
$$

where $\epsilon_{0}$ is the permittivity in free space.

The magnetic rigidity is determined by the momentum per charge, as follows,

$$
B \rho=3.3356 p_{c h}
$$

where the magnetic field $B$ is in Tesla, the bending radius $\rho$ is in meter, and $p_{c h}$ is in $G e V / c$.

For the continuity of energy and momentum at the charge exchange, units per nucleon are used. This is accomplished simply multiplying the unit per charge by a factor of $Q / A$. The longitudinal and transverse focusing is, however, still determined by the unit per charge.

In the following, we will go through with the longitudinal and transverse parameters and formulations.

\section{Longitudinal Formulations}

\subsection{General parameters}

The RF frequency is determined by,

$$
f_{R F}=\frac{h c^{2} p}{2 \pi R E}
$$

where $h$ is the harmonic number and $R$ is the machine radius. This equation comes from the relation between the particle momentum and energy,

$$
p=\frac{\beta E}{c}
$$

and because of the cancellation, any same set of units can be used.

The synchrotron oscillation frequency is derived from the relation between the phase deviation and the energy deviation, where the energy deviation is compensated by the RF voltage. Since this compensation is determined by the charges, therefore, the energy per charge should be used,

$$
\omega_{S}=\sqrt{\frac{-e V_{R F} \eta \omega_{R F}^{2} \cos \phi_{S}}{2 \pi \beta^{2} E_{c h} h}}
$$

The synchronous phase $\phi_{S}$ can be calculated in different ways. One way is to use $d B / d t$ as, 


$$
\phi_{S}=\sin ^{-1}\left(\frac{2 \pi R \rho}{V_{r f}} \frac{d B}{d t}\right)
$$

Considering the equation (1.7), in fact, the momentum per charge is used.

Assuming a smooth bunch, the momentum spread can be calculated,

$$
\frac{\Delta p}{p}= \pm \frac{\omega_{S} \phi_{\ell}}{|\eta| \omega_{R F}}
$$

where $\phi_{\ell}$ is the half bunch length in rad. Again, considering $\omega_{S}$ in (2.3), the unit per charge is used.

These parameters are the hard parameters, which can be physically verified. Some other parameters such as the bucket and bunch area, height, etc., are basically the convention, therefore the situation becomes more flexible and probably more subtle than the hard parameters.

\subsection{Bucket and bunch}

For simplicity, we consider the case of $\phi_{S}=0$. The most important parameter is the stationary bucket half height, which can be derived from a canonical coordinates of the phase space as,

$$
H_{B K S}=\frac{2 \beta^{2} E \omega_{S}}{|\eta| \omega_{R F}^{2}}
$$

The stationary bucket area is,

$$
A_{B K S}=8 H_{B K S}
$$

and the longitudinal emittance, i.e. the bunch area, is defined as,

$$
A_{B H}=\frac{\pi}{2} \phi_{\ell}^{2} H_{B K S}
$$

\subsection{Unit per charge and per nucleon}

One would like to have a constant longitudinal emittance through a change of charge state. For this purpose, the longitudinal emittance per nucleon is used, in $e V s / u$. The equation (2.6) shows that the bucket height per nucleon, calculated using energy per nucleon, is $Q / A$ times of the one per charge, given $\omega_{S}$ unchanged. Complication arises that $\omega_{S}$ must be calculated using $E_{c h}$, therefore, one needs to be careful using other formulations, which, on other hand, are always suitable for protons and electrons. We show two examples.

1. The the bucket height in [1] is,

$$
H_{B K S}=\sqrt{\frac{2 e V_{R F} \beta^{2} E}{\pi h|\eta| \omega_{R F}^{2}}}
$$


Note that here the difference between the bucket height per nucleon and per charge is $\sqrt{Q / A}$, this error comes from the improper cancellation of $E$, when substituting (2.3) into (2.6).

2. A further manipulation on (2.9), using (2.3), yields,

$$
H_{B K S}=\frac{e V_{R F}}{\pi h \omega_{S}}
$$

where exits no difference between which unit is used. The cause of the problem is also the improper cancellation of energy.

The straightforward way to evaluate the longitudinal emittance is to use the bunch length and the energy spread, as that in [2], which agrees with the use of (2.6) and (2.8). However, the complication of the change of focusing as charge state changes still needs to be resolved, which is the subject of following subsections.

\subsection{Matching with the same $\omega_{R F}$}

Whenever the charge state changes, the longitudinal focusing is changed. Let us recall the situation of the RF voltage variation. When the RF voltage changes, the stationary bucket height will change. Should the RF voltage change non-adiabatically, the longitudinal emittance would blow-up. If the $\mathrm{RF}$ voltage increases, then the bucket height also increases, therefore the particles at the large phase deviation contribute to the emittance blow-up. If the RF voltage decreases, then the particles with large energy deviation contribute to the emittance blow-up. For this reason, the adiabaticity in the RF voltage manipulation is required to conserve the longitudinal emittance, or bunch area, $A_{B H}$.

The change of heavy ion charge state, usually at the transfer lines, does not allow an adiabatic change. Thus, one needs to consider the matching of the focusing. The situation with the same $\omega_{R F}$ applies to the Booster to AGS transfer, provided that the following two important issues at the stripping are disregarded.

1. Emittance blow-up.

2. Energy loss.

Since the RF frequency is not changed, the bunch length $\phi_{\ell}$ keeps constant. Thus, the matching condition is that the stationary bucket height is constant, in the unit of $\mathrm{eVs} / \mathrm{u}$, according to (2.8). With this matching condition satisfied, the longitudinal emittance per nucleon would be conserved. Considering (2.6), given that $\beta, E_{u}$, and $\omega_{R F}$ are all the same, the matching condition is,

$$
\frac{\omega_{S 1}}{\left|\eta_{1}\right|}=\frac{\omega_{S 2}}{\left|\eta_{2}\right|}
$$

where the footnotes 1 and 2 represent that before and after the stripping. Using (2.3), the matching condition becomes, 


$$
\frac{V_{R F 1}}{E_{c h 1} h_{1}\left|\eta_{1}\right|}=\frac{V_{R F 2}}{E_{c h 2} h_{2}\left|\eta_{2}\right|}
$$

Taking the gold beam transfer from the Booster to the AGS as an example. The beam extracted from the Booster at $E_{u}=1.027 \mathrm{GeV} / u$, at the charge state $Q=32$, therefore, we have $E_{c h 1}=E_{u} \times A / Q=6.322 \mathrm{GeV}$. Other parameters are $V_{R F 1}=80 \mathrm{KV}, h_{1}=4$, and $\eta_{1}=-0.78$. In the BTA transfer line, the ions are stripped to $Q=77$, therefore, disregarding the energy loss, we have $E_{c h 2}=2.627 \mathrm{GeV}$. From the parameters $h_{2}=16$, and $\eta_{2}=-0.81$, we get the matching $R F$ voltage $V_{R F 2}=138 K V$.

\subsection{Matching with the different $\omega_{R F}$}

With different $R F$ frequencies, the bunch length in rad., i.e. $\phi_{\ell}$, would be different. This can be seen from the relation,

$$
\phi_{\ell}=\omega_{R F} \tau_{\ell}
$$

where $\tau_{\ell}$ is the half bunch length in sec., which is to be unchanged.

Again under the guideline of the constant longitudinal emittance per nucleon, we use (2.6) and (2.8), then the matching condition is,

$$
\frac{\omega_{S 1}}{\left|\eta_{1}\right|}=\frac{\omega_{S 2}}{\left|\eta_{2}\right|}
$$

where $\omega_{r f}^{2}$ in (2.6) is cancelled by the one included in $\phi_{\ell}^{2}$ of (2.8), see (2.13). Although this equation looks the same as (2.11), using (2.3), the real matching condition would be different from $(2.12)$,

$$
\frac{V_{R F 1} \omega_{R F 1}^{2}}{E_{c h 1} h_{1}\left|\eta_{1}\right|}=\frac{V_{R F 2} \omega_{R F 2}^{2}}{E_{c h 2} h_{2}\left|\eta_{2}\right|}
$$

this is because that $\omega_{R F}^{2}$ can no longer be cancelled from the two sides of the equation.

Let us take the gold beam transfer from the AGS to the RHIC as an example. Using the parameters given in [2], the beam extracted from the AGS at $E_{u}=11.73 \mathrm{GeV} / u$, with the charge state $Q=77$, therefore, we have $E_{c h 1}=E_{u} \times A / Q=30.01 \mathrm{GeV}$. Other parameters are $V_{R F 1}=320 \mathrm{KV}, h_{1}=12, \eta_{1}=-0.0074$, and $f_{R F 1}=4.44 \mathrm{MHz}$. The ions are stripped in the transfer line to $Q=79$, therefore, we have $E_{c h 2}=29.25 \mathrm{GeV}$. From the parameters $h_{2}=360, \eta_{2}=-0.0044$, and $f_{R F 2}=28.1 \mathrm{MHz}$, we get the matching $R F$ voltage $V_{R F 2}=139$ $K V$.

For another example, we consider the BTA transfer with energy loss. The beam momentum loss was found as $14 \mathrm{MeV} / \mathrm{c}$ per nucleon [3], about $3 \%$. Now we have $\beta=0.409$ instead of $\beta=0.421$ at the AGS end. The AGS RF frequency needs to be $f_{R F 2}=2.434 \mathrm{MHz}$, which is different from the Booster extraction $\mathrm{RF}$ frequency $f_{R F 1}=2.502 \mathrm{MHz}$. Therefore, the equation (2.15) is more adequate in this case for the matching. The other parameters at the AGS injection are $E_{c h 2}=2.611 \mathrm{GeV}$ and $\eta_{2}=-0.82$. Thus, for the same conditions at the Booster extraction, the matching RF voltage at the AGS becomes $V_{R F 2}=147 \mathrm{KV}$, about $6.5 \%$ higher. 
The matching condition with the different $\omega_{R F}$ is more general than the one of harmonic number change in the ring, which is obtained from (2.15) as,

$$
V_{R F 1} h_{1}=V_{R F 2} h_{2}
$$

\section{Longitudinal Instabilities}

In the longitudinal instability analysis, the longitudinal focusing plays a key role. Therefore, the unit per charge should be used. For instance, the bunched beam longitudinal microwave instability threshold is given in [4] as,

$$
\left|\sum_{n=-\infty}^{\infty} n Z_{L}(n)\right|<\frac{2 \omega_{S} \gamma(2 \pi R)^{2}}{N r_{0}|\eta| c^{2} \omega_{0}} \Delta \omega
$$

where $Z_{L}(n)$ is the longitudinal impedance. In the beam dynamic equation [5],

$$
\omega-m \omega_{S}=\frac{j 2 \pi m \omega_{S} I_{0}}{V_{R F} \cos \phi_{S}} \sum_{n=-\infty}^{\infty} \frac{Z_{L}(n)}{n} \Lambda_{1}^{2}(n)
$$

where $\Lambda_{1}^{2}(n)$ is the beam power spectrum, only hard parameters are used. Using (3.2), the equivalent equation to (3.1) is [6],

$$
\left|\sum_{n=-\infty}^{\infty} n Z_{L}(n)\right|<\frac{2 V_{R F}\left|\cos \phi_{S}\right|}{\omega_{S} I_{0}} \Delta \omega
$$

Considering (2.3), $r_{0, c h}$ should be used in (3.1).

Also in the Keil-Schnell criterion for the coasting beam [7],

$$
\left|\frac{Z_{L}(n)}{n}\right|<\frac{F \gamma E_{0}|\eta|}{e I_{0}}\left(\frac{\Delta p}{p}\right)^{2}
$$

where $F$ is a scaling factor, the rest energy per charge $E_{0, c h}$ should be used.

\section{Transverse Formulations}

The transverse normalized emittance, including $95 \%$ particles, is defined as,

$$
\epsilon_{N}^{95 \%}=\frac{6 \sigma^{2}}{\bar{\beta}(s)} \beta \gamma
$$

where $\bar{\beta}(s)=R / \nu$ is the average beta function. The conventional calculations for coherent and incoherent tune shift are

$$
\Delta \nu_{c o h}=\frac{-N r_{0} R}{4 \pi \nu_{0} B_{f} \beta^{2} \gamma^{3}} \frac{1}{b^{2}}
$$

where $b$ is the radius of the vacuum pipe, and 


$$
\Delta \nu_{i n c}=\frac{-N r_{0} R}{4 \pi \nu_{0} B_{f} \beta^{2} \gamma^{3}} \frac{1}{a^{2}}
$$

where $a$ is the beam size, it is $\sqrt{2}$ times of the rms beam size $\sigma$,

$$
a=\sqrt{2} \sigma
$$

We note that $r_{0, c h}$ should be used in (4.2) and (4.3).

\section{Transverse Instabilities}

The transverse instability problem can be solved similarly to the longitudinal counterpart. We may start from the beam dynamic equation [8],

$$
\omega-\omega_{\beta}-m \omega_{S}=\frac{j e I_{0}}{2 R m_{0} \gamma \omega_{\beta}} \sum_{n=-\infty}^{\infty} Z_{T}(n) \Lambda_{0}^{2}\left(n^{\prime}\right)
$$

where $m_{0, c h}$ should be used. The followings are examples in applying (5.1).

For bunched beams, the transverse microwave instability threshold is given in [4],

$$
\left|\sum_{n=-\infty}^{\infty} Z_{T}(n)\right|<\frac{2 \omega_{\beta} \gamma T_{0}^{2}}{N r_{0} c} \Delta \omega
$$

where $r_{0, c h}$ should be used. A more accurate formulation is give in [9],

$$
\left|\frac{\sum_{n=-\infty}^{\infty} Z_{T}(n) h_{0}\left(n^{\prime}\right)}{\sum_{n=-\infty}^{\infty} h_{0}\left(n^{\prime}\right)}\right|<\frac{2 \omega_{\beta} \gamma m_{0} z_{L}}{e I_{0}} \Delta \omega
$$

where $m_{0, c h}$ should be used. For coasting beams, the microwave criterion is given in [7],

$$
\left|Z_{T}(n)\right|<\frac{8 F E_{0} \gamma \omega_{\beta}}{e I_{0} R \omega_{0}^{2}} \Delta \omega
$$

where $E_{0, c h}$ should be used. 


\section{References}

[1] S. Ohnuma, 'The Beam and the Bucket,' Fermilab Report, TM-1381, 1986.

[2] RHIC Design Manual, BNL, 1996.

[3] C.J. Gardner, 'Heavy Ion Parameters for 1995,' AGS Tech. Note 417, 1995.

[4] A. Chao, Physics of Collective Beam Instabilities in High Energy Accelerators, Wiley, New York, 1994.

[5] S.Y. Zhang and W.T. Weng, 'On Solutions of Sacherer Integral Equation,' BNL60557, 1994.

[6] S.Y. Zhang, 'Microwave Instability Threshold,' PAC, Vancouver, B.C., 1997.

[7] W. Schnell, 'Coherent Instabilities of Proton Beam in Accelerator and Storage Rings Experimental Results, Diagnosis and Cures,' CERN 77-13, p.231, 1977.

[8] S.Y. Zhang, 'Transverse Bunched Beam Instability,' AGS Tech. Note 422, BNL, 1995.

[9] F. Sacherer, 'Transverse Bunched Beam Instabilities - Theory,' 9th Conf. High Energy Particle Accelerators, p.347, 1974. 